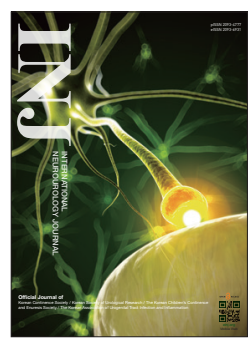

\title{
A Possible Pathogenic Linkage Among Headache, Migraine, and Nocturnal Enuresis in Children
}

\author{
Pietro Ferrara ${ }^{1,2}$, Livia Dell'Aquila ${ }^{2}$, Giacomo Perrone ${ }^{2}$, Giulia Spina ${ }^{2}$, Francesco Miconi² ${ }^{2}$, Valentina Rapaccini ${ }^{3}$, Ester Del Vescovo ${ }^{2}$, \\ Vincenzo Di Lazzaro ${ }^{3}$, Alberto Verrotti ${ }^{4}$ \\ ${ }^{1}$ Institute of Pediatrics, Catholic University Medical School, Rome, Italy \\ ${ }^{2}$ Service of Pediatrics Campus Bio-Medico University, Rome, Italy \\ ${ }^{3}$ Institute of Neurology, Campus Bio-Medico University, Rome, Italy \\ ${ }^{4}$ Department of Pediatrics, University of Perugia, Perugia, Italy
}

\begin{abstract}
Purpose: This study aims to evaluate the prevalence of headaches and migraine in children with nocturnal enuresis (NE) and to improve knowledge on these conditions. In particular, for this purpose, a possible pathogenic relationship linking both conditions and the impact of headaches and migraine on NE persistence was evaluated.

Methods: Researchers enrolled 123 children with NE, aged between 5 and 15 years, referred to the Service of Pediatrics, Campus Bio-Medico University Hospital of Rome between January 2014 and January 2015. Parents of all children enrolled in the study were invited to complete a self-reported questionnaire. The study protocol was approved by the Human Research Ethics Committee of Campus-Bio-Medico University. The NE group data was compared with the data of a control group (107 children).

Results: Of the eligible patients, 7.8\% suffer from headaches/migraine (mean age, 9.63 years; interquartile range [IQR], 3.5 years) and $47.1 \%$ have a family history of headaches (mean age, 8.46 years; IQR, 3.75 years). Of the 8 patients with headaches, all are male, 3 have tension-type headaches ( 2 of them have maternal family history) and 5 have migraine ( 3 of them have maternal family history). Of the $35.3 \%$ with a migraine family history (mean age, 8.36 years; IQR, 3.5 years), 22 are male, and 14 are female. Three of these patients have migraine. A total of $92.2 \%$ suffer from NE but not from headaches (mean age, 8.43 years; IQR, 3 years). Of these patients, 33 are female (35.1\%), and 61 are male (64.9\%). In the control group, 4.7\% (5 out of 107) of the children suffer from headaches, and of these, 4 are affected by nonmigraine headaches and 1 by migraine.

Conclusions: In conclusion, according to the hypothesis, NE and headaches/migraine could be linked by several similarities.
\end{abstract}

Keywords: Melatonin; Enuresis; Child

- Research Ethics: The study protocol was approved by the Human Research Ethics Committee of Campus-Bio-Medico University (approval number: 07.16.TS).

- Conflict of Interest: No potential conflict of interest relevant to this article was reported.

\section{INTRODUCTION}

Headache is a common complaint among children that occurs in $75 \%$ of adolescents and $25 \%$ of young children; it is also the most common neurological disease observed in clinical practice $[1,2]$. The International Headache Society classification system is distinguished into primary and secondary headache disorders [3]. In particular, migraine without aura and tension-type head-
Corresponding author: Pietro Ferrara (iD http://orcid.org/0000-0001-9449-3464 Institute of Pediatrics, Catholic University Medical School, L.go Francesco Vito, 1, 00168, Rome, Italy

E-mail pferrara@rm.unicatt.it, p.ferrara@unicampus.it / Tel: +39-06-30154348 / Fax: +39-06-3383211

Submitted: March 9, 2016 / Accepted after revision: March 30, 2016 
ache (TTH), both primary disorders, are the 2 most common types of headaches in children and adolescents. A migraine is a primary headache and has an important socioeconomic impact on children's and parents' quality of life and relationships [4]. The mean prevalence of (nonmigraine) headaches is 54.4\% (95\% confidence interval [CI], 43.1-65.8) and the mean prevalence of migraine is 9.1\% (95\% CI, 7.1-11.1) [5]. The World Health Organization recognized these disorders as a major public health concern, in particular by ranking migraine at 7th place among all worldwide diseases causing ictal disability [6]. For these reasons, the clinical approach to an infant with headache should be systematic and seek early recognition and management [7].

Migraine can be defined as paroxysmal disorder with a natural fluctuation between a low and high frequency pattern. Its etiology is complex and includes modifiable and nonmodifiable risk factors. It is a chronic, painful syndrome with aspects such as psychiatric comorbidity, decreased quality of life, and environmental factors that can all influence the success of its treatment. A high and increasing attack frequency can lead to 'chronic migraine' (CM), which then becomes resistant to acute as well as prophylactic migraine medications. $\mathrm{CM}$ is defined as having more than 15 headache days in a month, with at least half of these showing migraine features for a period of at least 3 months [8]. Considering TTH in children and adolescents, there is a wide range of prevalence [9]. Many children and adolescents exhibit an overlapping pattern of migraine and TTH [10]; moreover, by the end of the teenage years, the diagnosis of TTH may convert to migraine or vice versa [11].

Nocturnal enuresis (NE) is the most common developmental pediatric disorder. It can be defined as wetting while asleep after the age of 5 years. The estimated prevalence of NE is agerelated and highly variable because diagnostic criteria are heterogeneous [12]. According to the Diagnostic and Statistical Manual of Mental Disorders (DSM-5), NE is defined as repeated voiding of urine into bed or clothes while asleep in children older than 5 years.

NE occurs when there is poor arousal from sleep in response to a sensation of full bladder in association with an overactive bladder and an excess of nocturnal urine production. Other risk factors that are shown to be associated with NE include: delay in attaining bladder control, physiological factors such as constipation, fecal incontinence, daytime urinary incontinence (UI), caffeine consumption, sleep apnea, upper airway obstructive symptoms, lower socioeconomic status, and black race [13].

$\mathrm{NE}$ can be distinguished into 2 subgroups: primary and sec- ondary NE. A child with primary NE has never been continuously continent for more than 6 months; secondary NE relates to relapse after at least 6 months of dryness. Secondary NE is also associated with organic or psychological causes such as urine infection, sexual abuse, diabetes, obstructive sleep hypoventilation, neurogenic bladder, or externalizing disorders such as attentiondeficit/hyperactivity disorder or conduct disorder.

$\mathrm{NE}$, without daytime symptoms of bladder dysfunction, is called monosymptomatic NE (MNE), while NE with any daytime lower urinary tract symptoms is considered a non-MNE [14].

The aim of this study is to evaluate the prevalence of headache in children with NE and to increase knowledge on these 2 conditions. In particular, for this purpose, this study evaluated a possible pathogenic relation linking both conditions and the impact of headache on NE persistence.

\section{MATERIALS AND METHODS}

The present study is an observational study conducted on 123 children with NE, aged between 5 and 15 years, referred to the to the Service of Pediatrics, Campus Bio-Medico University Hospital of Rome between January 2014 and January 2015. The study protocol was approved by the Human Research Ethics Committee of Campus-Bio-Medico University (approval number: 07.16.TS). The community comparison group consisted of 107 children of the general population whose data were collected from family pediatricians in the Rome area.

Children and their families were asked to participate in the study at the end of the clinical evaluation and after obtaining informed consent. Parents were queried regarding their child's birth, past medical and family history, current medical conditions, and medications. The inclusion criterion was primary NE while exclusion criteria included secondary NE, urogenital malformations, renal failure, and diabetes. Parents of all children enrolled in the study were invited to complete a self-report questionnaire adapted from the CHILD/Adolescent Hardship questionnaire [15]. The information gathered through the 13tem questionnaire included 4 items regarding general data: name, surname, gender, age; 9 items concerning headaches: presence/absence of headaches in child, presence/absence of headaches among relatives, age of headache onset, daily/nightly symptoms, duration of each attack, pain characteristics (location, type, degree, associated symptoms), drugs used during the attack, relation with physical and daily activities, and relation with certain types of food. 


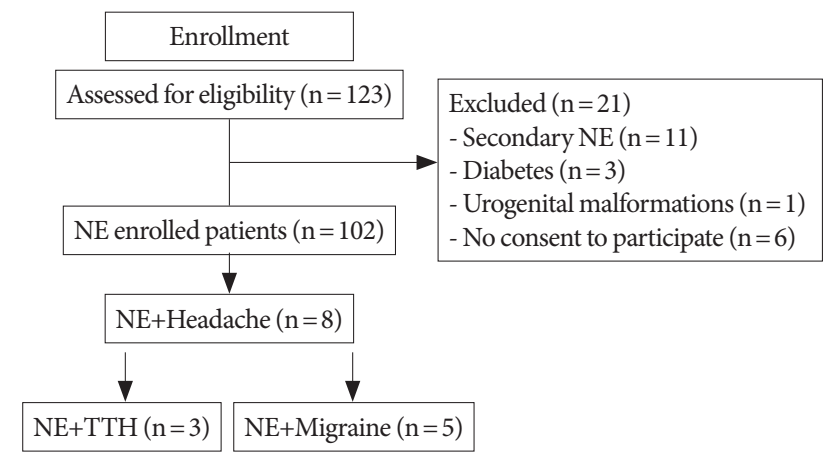

Fig. 1. CONSORT (consolidated standards for reporting of trials) flow diagram. NE, nocturnal enuresis; TTH, tension-type headaches.

\section{RESULTS}

The study population included 123 children with NE. Of these, 15 patients were excluded ( 11 for secondary NE, 3 for diabetes, 1 for urogenital malformations), and 6 patients refused to participate. Therefore, the study consisted of 102 children; 69 male (68\%) and 33 female (32\%), aged between 5 and 15 years (mean age, 8.52 years; interquartile range [IQR], 3 years). In particular, the male mean age was 1.75 years higher than the female mean age. The age in the comparison group was between 5 and 14 years (mean age, 8.19 years); $61 \%$ were male and $39 \%$ were female.

Of the 102 eligible patients, 8 (7.8\%) suffer from headaches (mean age, 9.63 years; IQR, 3.5 years) and $48(47.1 \%)$ have a family history of headaches (mean age, 8.46 years; IQR, 3.75 years). Of the 8 patients with headaches, all are male, 3 have TTH ( 2 of them have maternal family history) and 5 have migraine ( 3 of them have maternal family history) (Fig. 1, Table 1).

Considering family history, 33 patients (32.4\%) (12 female and 21 male patients) have a maternal family history of headaches (mean age, 8.21 years; IQR, 2.5 years), 19 children (18.6\%) ( 6 female and 13 male patients) have a paternal family history of headaches (mean age, 9.11 years; IQR, 4 years), and 6 patients (5.9\%) (2 female and 4 male patients) have grandparents suffering from headaches (mean age, 7.33 years; IQR, 3 years).

Of the 12 patients (11.8\%) with a family history of TTH (mean age, 8.75 years; IQR, 4.5 years), 8 are male and 4 are female patients. Two of these patients have TTH.

Of the 36 patients (35.3\%) with a family history of migraine (mean age, 8.36 years; IQR, 3.5 years), 22 are male and 14 are female patients. Three of these patients have migraine.

Ninety-four patients (92.2\%) suffer from NE but not from
Table 1. Number and sex of patients at different age

\begin{tabular}{llcc}
\hline Age group $(\mathrm{yr})$ & \multicolumn{1}{c}{ NE } & NE+Migraine & NE+TTH \\
\hline $5-7$ & $42($ male:female $=24: 18)$ & 2 & - \\
$8-10$ & $38($ male:female $=23: 15)$ & 1 & 2 \\
$11-14$ & $22($ male $=22)$ & 2 & 1 \\
\hline
\end{tabular}

NE, nocturnal enuresis; TTH, tension-type headaches.

headaches (mean age, 8.43 years; IQR, 3 years). Of these patients, 33 are female (35.1\%) and 61 are male patients (64.9\%).

In the control group, 5 out of 107 children (4.7\%) suffer from headaches; of these, 4 are affected by nonmigraine headaches and 1 by migraine.

\section{DISCUSSION}

Migraine shares striking similarities with NE. Both conditions are two common multifactorial pediatric disorders involving genetic, behavioral, and neurologic aspects with a complex pathophysiology which is still not completely known [16].

Studies have reported that children with primary NE and migraine might show dysfunction of the autonomic nervous system [17]. In particular, several studies observed sympathetic and parasympathetic nervous system hyperactivity in both enuretic and migraine children; moreover, Yerdelen et al. [18] demonstrated that the most common dysfunction in migraine is sympathetic hypofunction.

Considering anatomical aspects, neurological structures are thought to be involved in the pathogenesis of migraine and NE. Montagna [19] showed that the periaqueductal grey matter, the dorsal raphe nuclei and the locus coeruleus represent the "migraine generator"; furthermore, the locus coeruleus plays an important role in the activation of arousal circuits and it is also related to NE pathophysiology.

Some authors have confirmed the well-known relationship between sleep disorders and migraines: somnambulism, sleep terrors, and enuresis were observed in children affected by headaches $[20,21]$. This is probably based on common pathophysiological substrates [22]. In particular, the increased prevalence of migraine in patients with sleep disorders is associated with the hypothalamus regulation of circadian rhythm [23].

Clinical literature has demonstrated contrasting results regarding the onset of migraine or enuretic attacks during the night; in particular, micturition tends to occur during non-rapid-eye-movement sleep, while migraine attacks seem to be 
linked to rapid-eye-movement sleep stages [24,25]. Moreover, recent neurometabolic studies have reported altered levels of melatonin both in migraine sufferers and enuretics $[26,27]$.

Wöber-Bingöl [5] observed that the overall mean prevalence of nonmigraine headache is $54.4 \%$ ( $95 \%$ CI, $43.1-65.8$ ). Our data, in contrast with previous studies, showed that the overall prevalence of TTH is $2.9 \%$ (95\% CI, 0.6-8.3). This strong prevalence discordance is probably due to a different sample size considered (145,031 patients vs. 102 patients). Moreover, our prevalence is related only to TTH while Bingol is related to nonmigraine headaches (tensive headache, trigeminal autonomic cephalgia, cluster headache, paroxysmal hemicranias, hypnic headache, exertional headaches, primary stabbing headache, new daily persistent headache, and others) [28].

In addition, our results could have underestimated the prevalence of headache in enuretic children because of the difficulty in investigating cephalgic symptoms, especially in very young children who may be incapable of describing adequately the headache characteristics to an examiner [29]. Furthermore, the description of the quality and severity of the pain could be difficult to analyze. For these reasons, some authors suggest the use of an age-appropriate scale [2]. Moreover, our data mainly refer to a population of children under the age of 10 , and case-studies of the prevalence of headaches in the literature generally report data referring to an adolescent population.

On the contrary, the prevalence of migraine in our study did not show significant differences with the general population; in fact, the prevalence of $4.9 \%$ ( $95 \%$ CI, 9.6-11) (5 of 102 patients) is not different from the general population $(9.1 \% \mathrm{CI}, 7.1-11.1)$ [5].

Epidemiological studies showed that NE represents a greater risk factor for the onset of headaches, especially in females [30]. Moreover, Bigal et al. [31] revealed that females are more commonly affected with all types of headaches. In our study, by contrast, headaches affected only male children. This may be due to the fact that our study enrolled patients affected by NE; with respect to sex, this disorder is significantly higher in males compared to females. The ratio of the incidence of NE reported in the literature in boys vs. girls is 2:1, and within our data, the ratio is the same [32].

In particular, Butler et al. [33] observed that males are more often diagnosed with NE. Despite this observation, our study found that there is a significant correlation between maternal headache and NE in children. In particular, of a total of 36 patients with a family history of migraine, 23 mothers have ceph- algic symptoms (23 of 36 [64\%]; odds ratio, 5.78).

Furthermore, comparing the results of the NE group with the data of the control group, it was observed that the prevalence of headache in the NE group (7.8\%) is higher than the prevalence of headache in the control group. Specifically, in the NE group, the number of children with migraine (5 out of 8 of those children with headache) is higher than the number of children with migraine ( 1 out of 5 of those children with headache) in the control group.

Finally, migraine and NE also share psychological factors: they are both associated with high incidences of internalizing problems such as anxiety and depression that negatively impact quality of life $[34,35]$.

In conclusion, according to our hypothesis, NE and headaches/migraine could be linked by several similarities. Further studies are necessary to elucidate the relationship between headaches and NE and between family history of headache and NE. In particular, additional studies might address the issue of analyzing headache prevalence considering a bigger sample size and a long follow-up period evaluation. It could also be interesting to assess the impact of NE treatment on cephalgic symptoms. Moreover, validated scales should be used to diagnose headaches in young children and also to distinguish migraine and nonmigraine headaches in order to highlight all of the specific features.

\section{REFERENCES}

1. Bille BS. Migraine in school children. A study of the incidence and short-term prognosis, and a clinical, psychological and electroencephalographic comparison between children with migraine and matched controls. Acta Paediatr Suppl 1962;136:1-151.

2. Kacperski J, Kabbouche MA, O’Brien HL, Weberding JL. The optimal management of headaches in children and adolescents. Ther Adv Neurol Disord 2016;9:53-68.

3. Ozge A, Termine C, Antonaci F, Natriashvili S, Guidetti V, WöberBingöl C. Overview of diagnosis and management of paediatric headache. Part I: diagnosis. J Headache Pain 2011;12:13-23.

4. Martelletti P, Katsarava Z, Lampl C, Magis D, Bendtsen L, Negro A, et al. Refractory chronic migraine: a consensus statement on clinical definition from the European Headache Federation. J Headache Pain 2014;15:47.

5. Wöber-Bingöl C. Epidemiology of migraine and headache in children and adolescents. Curr Pain Headache Rep 2013;17:341.

6. Vos T, Flaxman AD, Naghavi M, Lozano R, Michaud C, Ezzati M, et al. Years lived with disability (YLDs) for 1160 sequelae of 289 
diseases and injuries 1990-2010: a systematic analysis for the Global Burden of Disease Study 2010. Lancet 2012;380:2163-96.

7. Stang PE, Osterhaus JT. Impact of migraine in the United States: data from the National Health Interview Survey. Headache 1993; 33:29-35.

8. Özge A, Yalin OÖ. Chronic migraine in children and adolescents. Curr Pain Headache Rep 2016;20:14.

9. Anttila P. Tension-type headache in childhood and adolescence. Lancet Neurol 2006;5:268-74.

10. Monteith TS, Sprenger T. Tension type headache in adolescence and childhood: where are we now? Curr Pain Headache Rep 2010; 14:424-30.

11. Karli N, Bican A, Zarifoğlu M. Course of adolescent headache: 4-year annual face-to-face follow-up study. J Headache Pain 2010; 11:327-34.

12. Chiozza ML, Bernardinelli L, Caione P, Del Gado R, Ferrara P, Giorgi PL, et al. An Italian epidemiological multicentre study of nocturnal enuresis. Br J Urol 1998;81 Suppl 3:86-9.

13. Ferrara P, De Angelis MC, Caporale O, Malamisura M, Del Volgo $\mathrm{V}$, Vena F, et al. Possible impact of comorbid conditions on the persistence of nocturnal enuresis: results of a long-term follow-up study. Urol J 2014;11:1777-82.

14. Harari MD. Nocturnal enuresis. J Paediatr Child Health 2013;49:26471.

15. Wöber-Bingöl Ç, Wöber C, Uluduz D, Uygunoğlu U, Aslan TS, Kernmayer M, et al. The global burden of headache in children and adolescents - developing a questionnaire and methodology for a global study. J Headache Pain 2014;15:86.

16. Chang SS, Ng CF, Wong SN; Hong Kong Childhood Enuresis Study Group. Behavioural problems in children and parenting stress associated with primary nocturnal enuresis in Hong Kong. Acta Paediatr 2002;91:475-9.

17. Järvelin MR, Moilanen I, Kangas P, Moring K, Vikeväinen-Tervonen $\mathrm{L}$, Huttunen NP, et al. Aetiological and precipitating factors for childhood enuresis. Acta Paediatr Scand 1991;80:361-9.

18. Yerdelen D, Acil T, Goksel B, Karatas M. Heart rate recovery in migraine and tension-type headache. Headache 2008;48:221-5.

19. Montagna P. Hypothalamus, sleep and headaches. Neurol Sci 2006;27 Suppl 2:S138-43.

20. De Giorgis G, Miletto R, Iannuccelli M, Camuffo M, Scerni S. Headache in association with sleep disorders in children: a psychodiagnostic evaluation and controlled clinical study--L-5-HTP versus placebo. Drugs Exp Clin Res 1987;13:425-33.

21. Bruni O, Fabrizi P, Ottaviano S, Cortesi F, Giannotti F, Guidetti V. Prevalence of sleep disorders in childhood and adolescence with headache: a case-control study. Cephalalgia 1997;17:492-8.

22. Lin J, Rodrigues Masruha M, Prieto Peres MF, Cianciarullo Minett TS, de Souza Vitalle MS, Amado Scerni D, et al. Nocturnal enuresis antecedent is common in adolescents with migraine. Eur Neurol 2012;67:354-9.

23. Casez O, Dananchet Y, Besson G. Migraine and somnambulism. Neurology 2005;65:1334-5.

24. Reimão R, Pachelli LC, Carneiro R, Faiwichow G. Primary sleep enuresis in childhood. Polysomnographic evidences of sleep stage and time modulation. Arq Neuropsiquiatr 1993;51:41-5.

25. Nørgaard JP, Hansen JH, Nielsen JB, Rittig S, Djurhuus JC. Nocturnal studies in enuretics. A polygraphic study of sleep-EEG and bladder activity. Scand J Urol Nephrol Suppl 1989;125:73-8.

26. Göder R, Fritzer G, Kapsokalyvas A, Kropp P, Niederberger U, Strenge $\mathrm{H}$, et al. Polysomnographic findings in nights preceding a migraine attack. Cephalalgia 2001;21:31-7.

27. Nevéus T, Stenberg A, Läckgren G, Tuvemo T, Hetta J. Sleep of children with enuresis: a polysomnographic study. Pediatrics 1999; 103(6 Pt 1):1193-7.

28. Headache Classification Committee of the International Headache Society (IHS). The International Classification of Headache Disorders, 3rd edition (beta version). Cephalalgia 2013;33:629-808.

29. Parisi P, Vanacore N, Belcastro V, Carotenuto M, Del Giudice E, Mariani R, et al. Clinical guidelines in pediatric headache: evaluation of quality using the AGREE II instrument. J Headache Pain 2014;15:57.

30. Di Prospero S, Fadda G, Gallo M. Enuresis: clinico-epidemiologic study. Preliminary communication: its relation to essential headache. Minerva Med 1986;77:1421-6.

31. Bigal ME, Lipton RB, Winner P, Reed ML, Diamond S, Stewart WF, et al. Migraine in adolescents: association with socioeconomic status and family history. Neurology 2007;69:16-25.

32. Ozkan S, Durukan E, Iseri E, Gürocak S, Maral I, Ali Bumin M. Prevalence and risk factors of monosymptomatic nocturnal enuresis in Turkish children. Indian J Urol 2010;26:200-5.

33. Butler RJ, Golding J, Northstone K; ALSPAC Study Team. Nocturnal enuresis at 7.5 years old: prevalence and analysis of clinical signs. BJU Int 2005;96:404-10.

34. Ferrara P, Vitelli O, Bottaro G, Gatto A, Liberatore P, Binetti P, et al. Factitious disorders and Munchausen syndrome: the tip of the iceberg. J Child Health Care 2013;17:366-74.

35. Ferrara P, Ianniello F, Romani L, Fabrizio GC, Gatto A, Chiaretti A. Five years of experience in nocturnal enuresis and urinary incontinence in children: where we are and where we are going. Urol Int 2014;92:223-9. 